\title{
A COMPARISON OF BOOTSTRAP AND MONTE CARLO APPROACHES TO TESTING FOR SYMMETRY IN THE HOUCK'S MODEL
}

\author{
Henry de-Graft Acquah, Senior Lecturer \\ Department of Agricultural Economics and Extension, University of Cape Coast, Ghana \\ E-mail: henrydegraftacquah@yahoo.com
}

\begin{abstract}
The power of the Houck's model of asymmetry is examined via bootstrap and Monte Carlo techniques. The results of bootstrap and Monte Carlo simulations indicate that the power of the Houck's test for asymmetry depends on sample size, level of asymmetry and the amount of noise in the data generating process. Furthermore, the simulation results suggest that both bootstrap and Monte Carlo methods are effective in rejecting the false null hypothesis of symmetric adjustment in large samples with small error size and strong levels of asymmetry. However, in small samples, with large error size and subtle levels of asymmetry, the results suggest that asymmetry test based on bootstrap are powerful than those based on the Monte Carlo methods. I conclude that both bootstrap and Monte Carlo algorithms provide useful tools for investigating the power of the test of asymmetry.
\end{abstract}

\section{KEY WORDS}

Monte Carlo Simulation; Bootstrap Methods; Houck's Model; Power Test; Asymmetry.

Houck (1977) proposes a methodology to investigate asymmetric adjustments in price transmission processes. This involves specifying asymmetries to affect the direct impact of price increases and decreases and does not take into account adjustments to equilibrium level.

Some studies (Capps and Sherwell, 2007) have applied this methodology to a series of problems and note the inabilities of the Houck's model to detect asymmetries in practice. Against this background, Acquah 2010 investigated the power of the Houck's model via Monte Carlo experimentation and finds that the Houck's model has low power in rejecting the null of symmetric adjustments. Alternatively, Acquah 2013 using bootstrap simulations demonstrated that the Houck's model has low power in bootstrap samples.

Though Acquah (2010, and 2013) sheds light on the low power of the Houck's model via bootstrap and Monte Carlo simulations, these previous studies fails to provide a comparison of the bootstrap and Monte Carlo methods in the power analysis test. A fundamental question such as, under what conditions will bootstrap and Monte Carlo techniques of testing for asymmetry lead to the same results remains unanswered. Empirically, this study fills a gap in the literature by providing a comparison of asymmetry test based on bootstrap with those based on Monte Carlo methods.

The purpose of this study is to support the claim that the failure of the Houck's model to capture asymmetry in practice is due to low power and in so doing provide a comparison of bootstrap and Monte Carlo based test for asymmetry in the Houck's model.

The paper is organized as follows. The introductory section is followed by sections on measuring asymmetry, testing for symmetry in the Houck's model, Monte Carlo experiments and bootstrap methods. This is followed by the results and discussion and the conclusions of the study.

Measuring Asymmetry. Tweenten and Quance (1969) were the first to develop a model to test for asymmetric price transmission. This approach of testing for asymmetry was later modified by Wolffram (1971).Wolffram's approach to measuring asymmetry was further modified by Houck (1977). Houck's contribution which is most widely cited involves developing a more rigorous approach to specifying and testing non reversible linear functions in economic research. Houck's paper stimulated considerable interest in the study of price asymmetry. For instance Ward (1982) extended Houck's specification by including lags. Boyd and Brorsen (1998) were the first to employ lags to differentiate between magnitude and speed of transmission. However, the Houck's model has been found to have low power (Capps and Sherwell, 2007, Acquah, 2010).

Testing for Asymmetry in the Houck's Model. Houck (1977) model of testing for asymmetric price transmission can be specified as: 


$$
\Delta \mathrm{y}_{t}=\beta_{1}^{+} \Delta x_{t}^{+}+\beta_{1}^{-} \Delta x_{t}^{-}+\varepsilon \quad \varepsilon \sim N\left(0, \sigma_{\varepsilon}^{2}\right)
$$

where $\Delta x_{t}^{+}$and $\Delta x_{t}^{-}$are the positive and negative changes in $x_{t}$. The independent variable

$x_{t}$ is generated as independent draws from normal distribution with a constant mean and a variance of one. Asymmetry is introduced by allowing differing speeds of adjustments for the coefficients of $\Delta x_{t}^{+}$and $\Delta x_{t}^{-}$in equation (1) and $\varepsilon$ is generated as i.i.d. draws from the standard normal distribution with a sample size $\mathrm{n}$. $\Delta \mathrm{y}_{t}$ is obtained using the values for beta, positive and negative changes in $x_{t}$ (i.e. $\Delta x_{t}^{+}$and $\Delta x_{t}^{-}$) and the error term as specified in equation 1 . Symmetric price transmission is tested by determining whether the coefficients ( $\beta_{1}^{+}$and $\beta_{1}^{-}$) are identical (i.e. $H_{0}: \beta_{1}^{+}=\beta_{1}^{-}$).

Monte Carlo Experiments. A Monte Carlo experiment involves the following steps. Consider the following linear regression:

$$
y=\beta_{0}+\beta_{1} x_{1}+\beta_{2} x_{2}+\varepsilon
$$

First, draw the exogenous variables of the model from their respective distributions. Second, draw a random sample of the error term in the model from its respective probability distribution function. Assuming values for the true parameters and drawing values of the stochastic element, we can estimate the endogenous variable and calculate the estimate of interest. Fundamentally, in Monte Carlo experiments, we draw from a specified distribution, by a random number generator. In effect, we make assumptions about the distributions and about the true values of parameters.

The Bootstrap. Bootstrap is a method to derive properties of the sample distribution of estimators. Efron and Tibshirani (1993) notes that bootstrap involves drawing with replacement from the original sample to produce samples of the same size as the original sample referred to as bootstrap sample. It therefore takes the empirical distribution function as the true distribution function. The great advantage compared with Monte Carlo methods is that we neither make an assumption about the distributions nor about the true values of the parameter.

Parametric Bootstrap. Parametric Bootstrap denotes the process of resampling from the residuals of a parametric regression model. It begins by estimating the model of interest and saving the residuals. It performs a simulation using the estimated parameter values as the true parameter values and the actual values of the explanatory variable as the fixed explanatory variable values. During this simulation study, errors are drawn with replacement from the set of original residuals. This residual based technique is referred to as parametric bootstrapping.

\section{RESULTS AND DISCUSSION}

In order to investigate the power of the test for asymmetry under various conditions, a series of bootstrap and Monte Carlo comparison of the Houck's model is carried out based on 10000 replications. In particular, the power of the Houck's model is examined under conditions of different sample sizes, noise levels and two levels of asymmetry given by

$$
\left(\beta_{2}{ }^{+}, \beta_{2}^{-}\right) \in(0.50,0.25) \operatorname{or}(0.75,0.25)
$$

We incorporate subtle and strong levels of asymmetry in the data generating process. The Houck's model is evaluated in terms of its ability to reject the incorrect null of symmetric adjustment using an F-test of the restricted versus the unrestricted model. The results in Table 1 and 2 indicate the low power of the conventional F-test in rejecting the incorrect null hypothesis of symmetry.

Table 1 - Rejection frequencies based on 10000 Monte Carlo Replications 


\begin{tabular}{|c|c|c|c|c|c|}
\hline $\begin{array}{l}\text { Sample } \\
\text { Size }\end{array}$ & $\left(\beta_{1}^{+}, \beta_{1}^{-}\right)$ & $\begin{array}{c}\text { Error } \\
\text { Size }(\sigma)\end{array}$ & $\bar{F}$ & $\operatorname{Rej}(5 \%)$ & $\operatorname{Rej}(1 \%)$ \\
\hline 50 & $(0.50,0.25)$ & 3 & 1.1981 & 0.0689 & 0.0157 \\
\hline 50 & $(0.50,0.25)$ & 2 & 1.4260 & 0.0924 & 0.0240 \\
\hline 50 & $(0.50,0.25)$ & 1 & 2.5709 & 0.2249 & 0.0804 \\
\hline 150 & $(0.50,0.25)$ & 3 & 1.5301 & 0.1113 & 0.0323 \\
\hline 150 & $(0.50,0.25)$ & 2 & 2.2117 & 0.1878 & 0.0675 \\
\hline 150 & $(0.50,0.25)$ & 1 & 5.6800 & 0.5696 & 0.3206 \\
\hline 500 & $(0.50,0.25)$ & 3 & 1.8699 & 0.1544 & 0.0508 \\
\hline 500 & $(0.50,0.25)$ & 2 & 4.9340 & 0.5122 & 0.2705 \\
\hline 500 & $(0.50,0.25)$ & 1 & 16.6603 & 0.9754 & 0.9132 \\
\hline 50 & $(0.75,0.25)$ & 3 & 1.7428 & 0.1271 & 0.0384 \\
\hline 50 & $(0.75,0.25)$ & 2 & 2.6578 & 0.2305 & 0.0824 \\
\hline 50 & $(0.75,0.25)$ & 1 & 7.5351 & 0.6630 & 0.4100 \\
\hline 150 & $(0.75,0.25)$ & 3 & 2.0589 & 0.1728 & 0.0554 \\
\hline 150 & $(0.75,0.25)$ & 2 & 5.7735 & 0.5732 & 0.3297 \\
\hline 150 & $(0.75,0.25)$ & 1 & 19.7038 & 0.9845 & 0.9421 \\
\hline 500 & $(0.75,0.25)$ & 3 & 7.9174 & 0.7450 & 0.5194 \\
\hline 500 & $(0.75,0.25)$ & 2 & 16.5895 & 0.9757 & 0.9126 \\
\hline 500 & $(0.75,0.25)$ & 1 & 63.4595 & 1.0000 & 1.0000 \\
\hline
\end{tabular}

Table 2 - Rejection frequencies based on 10000 Bootstrap Replications

\begin{tabular}{|c|c|c|c|c|c|}
\hline $\begin{array}{c}\text { Sample } \\
\text { Size }\end{array}$ & $\left(\beta_{1}^{+}, \beta_{1}^{-}\right)$ & $\begin{array}{c}\text { Error } \\
\text { Size }(\sigma)\end{array}$ & $\bar{F}$ & $\operatorname{Rej}(5 \%)$ & $\operatorname{Rej}(1 \%)$ \\
\hline 50 & $(0.50,0.25)$ & $3^{2}$ & 18956 & 0.1395 & 0.0512 \\
\hline 50 & $(0.50,0.25)$ & 2 & 2.1373 & 0.1664 & 0.0655 \\
\hline 50 & $(0.50,0.25)$ & 1 & 3.4550 & 0.2964 & 0.1431 \\
\hline 150 & $(0.50,0.25)$ & 3 & 2.1569 & 0.1805 & 0.0761 \\
\hline 150 & $(0.50,0.25)$ & 2 & 2.8612 & 0.2508 & 0.1202 \\
\hline 150 & $(0.50,0.25)$ & 1 & 6.3213 & 0.5546 & 0.3609 \\
\hline 500 & $(0.50,0.25)$ & 3 & 2.4825 & 0.2240 & 0.0967 \\
\hline 500 & $(0.50,0.25)$ & 2 & 5.6226 & 0.5025 & 0.3197 \\
\hline 500 & $(0.50,0.25)$ & 1 & 17.3154 & 0.9322 & 0.8489 \\
\hline 50 & $(0.75,0.25)$ & 3 & 2.4688 & 0.2003 & 0.0865 \\
\hline 50 & $(0.75,0.25)$ & 2 & 3.4056 & 0.2902 & 0.1466 \\
\hline 50 & $(0.75,0.25)$ & 1 & 8.2264 & 0.6190 & 0.4390 \\
\hline 150 & $(0.75,0.25)$ & 3 & 2.6982 & 0.2411 & 0.1085 \\
\hline 150 & $(0.75,0.25)$ & 2 & 6.5265 & 0.5625 & 0.3710 \\
\hline 150 & $(0.75,0.25)$ & 1 & 20.9906 & 0.9595 & 0.9036 \\
\hline 500 & $(0.75,0.25)$ & 3 & 8.5424 & 0.6936 & 0.5109 \\
\hline 500 & $(0.75,0.25)$ & 2 & 17.3892 & 0.9390 & 0.8510 \\
\hline 500 & $(0.75,0.25)$ & 1 & 64.7213 & 1.0000 & 1.0000 \\
\hline
\end{tabular}

Specifically, the Monte Carlo and bootstrap simulations indicate the low power of the conventional F-test in rejecting the null of symmetric adjustment in small sample sizes. For example in small samples with large error size and subtle level of asymmetry, Monte Carlo method achieved a rejection frequency of $7 \%$ compared to $14 \%$ for bootstrap at the $5 \%$ significance level as illustrated in the top parts of Tables 1 and 2. There is some increase in power when the amount of noise in the data generating process (DGP) is decreased systematically. Similarly, when the difference in asymmetric adjustment parameters is increased from 0.25 to 0.50 in the true model, an increase in power is also observed in Houck's test for asymmetry based on bootstrap and Monte Carlo methods as illustrated in Table 1 and 2. However, it is only when the sample size is increased to 500 that a reasonable results is obtained. For example, both the Bootstrap and Monte Carlo methods achieve a rejection frequency of $100 \%$ with regards to the rejection of the incorrect null hypothesis of symmetric adjustments at the $5 \%$ and $1 \%$ significance level as illustrated in the bottom parts of Tables 1 and 2 .

In summary, the sample size, difference between the asymmetric adjustment parameters and the amount of noise in the data generating process are important in the power of the test for asymmetry based on bootstrap and Monte Carlo methods. With large sample size or small noise, the Houck's model display greater power in rejecting the (false) null hypothesis of symmetry. 
The power of Houck's approach of detecting asymmetry has been evaluated using bootstrap and Monte Carlo methods. The results of the bootstrap and Monte Carlo simulations indicates that the power of the Houck's model depends on various conditions or design characteristics such as sample size, error size and the level of asymmetry. Rejection frequencies increase with increases in sample size, increases with increase in difference between the asymmetric adjustment speeds and increases with a decrease in the amount of noise in the true data generating process used in the application. The power of the test for asymmetry based on Monte Carlo and bootstrap methods are the same (have rejecting frequency of $100 \%$ ) if the sample size is large with a small error size and strong level of asymmetry. However, in small samples with large error size and subtle level of asymmetry, the test for asymmetry based on bootstrap outperforms the Monte Carlo approach, though both display low power. The low power of the Houck's model in rejecting the null of symmetric adjustment in the Monte Carlo and bootstrap simulations provides an explanation for the failure of the Houck model to capture asymmetric behaviour in practice. I conclude that both bootstrap and Monte Carlo algorithms provide useful tools for investigating the power of the test of asymmetry.

\section{REFERENCES}

[1] Acquah, H.D. (2013). A Bootstrap Approach to Evaluating the Power of the Houck's Test for Asymmetry in Journal of Social and Development Sciences, Vol. 4, No. 2 pp 69-73.

[2] Acquah, H. D. (2010): Testing for Symmetry in the Houck's Model in Indian Development Review: An International Journal of Development Economics -Vol. 8, No. 1, (January-December, 2010): 105-107.

[3] Boyd, M. S. and Brorsen, B. W. (1998). Price Asymmetry in the US Pork Marketing Channel, North Central Journal of Agricultural Economics, 10, pp.103- 109.

[4] Capps, O. and Sherwell, P. (2007). Alternative approaches in detecting asymmetry in farm-retail prices transmission of fluid milk. Journal of Agribusiness, vol 23 (3): 313331.

[5] Efron, B. \& Tibshirani, R. (1993). An Introduction to the Bootstrap, London: Chapman and Hall.

[6] Houck, J.P. (1977). An Approach to Specifying and Estimating Nonreversible Functions. American Journal of Agricultural Economics, vol 59: 570-572.

[7] Tweenten, L.G. and Quance, C.L. (1969). Positivistic Measures of aggregate Supply Elasticities: Some new Approaches. American Journal of Agricultural Economics, 51, pp. 342-352.

[8] Ward, R.W. (1982). Asymmetry in Retail, Wholesale and Shipping Point Pricing for fresh Vegetables. American Journal of Agricultural Economics, 62, pp. 205-212.

[9] Wolffram, R. (1971). "Positivistic Measures of Aggregate Supply Elasticities - Some New Approaches - Some Critical Notes," American journal of Agricultural Economics, 53, pp. 356-356. 